\title{
Two-Dimensional Impact Reconstruction Method for Rail Defect Inspection
}

\author{
Jie Zhao, ${ }^{1,2}$ Jianhui Lin, ${ }^{1}$ Jinbao Yao, ${ }^{3}$ and Jianming Ding ${ }^{1}$ \\ ${ }^{1}$ State Key Laboratory of Traction Power, Southwest Jiaotong University, Chengdu 610031, China \\ ${ }^{2}$ Department of Industry Manufacture, Chengdu University, Chengdu 610106, China \\ ${ }^{3}$ State Key Laboratory of Mechanical Transmission, Chongqing University, Chongqing 400030, China
}

Correspondence should be addressed to Jie Zhao; zhaojie0111@163.com

Received 2 May 2014; Accepted 27 June 2014; Published 17 July 2014

Academic Editor: Xuefeng Chen

Copyright (c) 2014 Jie Zhao et al. This is an open access article distributed under the Creative Commons Attribution License, which permits unrestricted use, distribution, and reproduction in any medium, provided the original work is properly cited.

\begin{abstract}
The safety of train operating is seriously menaced by the rail defects, so it is of great significance to inspect rail defects dynamically while the train is operating. This paper presents a two-dimensional impact reconstruction method to realize the on-line inspection of rail defects. The proposed method utilizes preprocessing technology to convert time domain vertical vibration signals acquired by wireless sensor network to space signals. The modern time-frequency analysis method is improved to reconstruct the obtained multisensor information. Then, the image fusion processing technology based on spectrum threshold processing and node color labeling is proposed to reduce the noise, and blank the periodic impact signal caused by rail joints and locomotive running gear. This method can convert the aperiodic impact signals caused by rail defects to partial periodic impact signals, and locate the rail defects. An application indicates that the two-dimensional impact reconstruction method could display the impact caused by rail defects obviously, and is an effective on-line rail defects inspection method.
\end{abstract}

\section{Introduction}

After the railroad was put into formal operation, the rail track inspection and maintenance became the key factor directly relating to the train operating safety. As the rail is used for long, some rail defects such as irregularity, fatigue crack, and fissure and pitting corrosion will appear $[1,2]$. With increased traffic at higher speed, the rail defects will lead to fiercer vertical vibration impact of the vehicle-track coupling system [3-5], which may cause frequent failures of train and tracks, or even occurrence of fatal accidents. Therefore, from the perspectives of train operating safety and the efficiency of inspection and maintenance, it is necessary to investigate the on-line inspection methods for rail defects.

The traditional rail inspection techniques mainly include the magnetic particle inspection method and electromagnetic inspection method, which use the specific railway inspection vehicle to detect rail defects. These methods are of low efficiency and high cost and are unable to achieve on-line inspection while the train is operating. The current inspection methods for rail detects are primarily the image inspection method [6-9], ultrasonic inspection method [10, 11], signal processing method [12-16], and so on. The image inspection method is to detect the defects by analyzing the acquired images of the rail surface. Being direct and reliable, but due to large number of data acquired and slow processing speed, this method is unsuitable for full coverage inspection on the railroad track. The ultrasonic inspection refers to the inspection of rail defects by transmitting ultrasound to the rail and using the receiver to receive the reflected or diffused ultrasonic energy. This technique can detect fast but has high requirement for accuracy in equipment installation and the equipment would be easily interfered with high false alarm probability when the train moves. And the signal processing method finds out and locates the rail defects through making analysis of the vertical vibration signals of the vehicle-track 
coupling system. It has the advantages of the abovementioned two techniques and can achieve the on-line inspection, so it becomes a focus for research.

Traditional signal processing methods are used to analyze the steady or approximate steady-state signals, for example, time domain statistics analysis, spectrum analysis, correlation analysis, and higher order spectral analysis; however, they are not applicable to analysis of the non-Gaussian and nonstationary signals caused by vertical vibration in vehicletrack coupling system. Modern signal processing methods such as short-time Fourier transform, wavelet transform, and empirical mode decomposition are well capable of analyzing the abovementioned signals but have unavoidable problems like difficulty in distinguishing the impacts caused by noise, aperiodic impact caused by train, and aperiodic impact caused by track.

After studying the wireless sensor network for the online inspection of rail defects, a two-dimensional impact reconstruction method is proposed in which multisensor information is taken as research target, analyzed and processed by improved modern time-frequency analysis method, and the image fusion processing technology is used to blank the periodic impacts caused by the defects of locomotive running gear or rail joints in the time-frequency spectrum of vertical impact signals, highlight and extract the impacts caused by rail defects, and realize the on-line inspection of rail defects.

\section{Wireless Sensor Network for On-Line Inspection of Rail Defects}

According to the actual distribution of train monitoring points, the wireless sensor network technology widely used in environmental monitoring [17] and military scouting [18] is introduced to the rail defect inspection. A wireless sensor network is constructed for on-line inspection of rail defects and realizes the real-time monitoring, sensing, and acquisition of vertical vibration signals of trains.

The sensor node distribution for rail defect inspection is designed as shown in Figure 1. The sensor at each node chooses the vibration impact sensor vertically installed above the axle box of the train bogie for monitoring the vertical vibration impact mainly resulting from track, wheels and transmission pair, and so forth.

All monitoring points distributed in Figure 1 may fully cover all monitored carriages. The vibration impact caused by the transmission failure and the flaws in wheels can transmit from axle, bearing, and axle box to the sensor node, and that caused by rail defects may also transmit to the sensor node through wheel, axle, and bearing and axle box. Besides, this distribution plan may enable each sensor node to gather the strong coupling data distinguishing wheel defect, transmission pair failure, and rail defect.

Since there are differences and certain coupling in the multisensor information collected by the wireless sensor network for the on-line inspection of rail defects, in order to make better fusion of the multisensor information, the monitoring points of all carriages at the same position will
TABLE 1: Signal group classification.

\begin{tabular}{lc}
\hline Group number & Left side node number/right side node number \\
\hline$G_{L 1} / G_{R 1}$ & $L_{1}, L_{5}, \ldots, L_{N-3} / R_{1}, R_{5}, \ldots, R_{N-3}$ \\
$G_{L 2} / G_{R 2}$ & $L_{2}, L_{6}, \ldots, L_{N-2} / R_{2}, R_{6}, \ldots, R_{N-2}$ \\
$G_{L 3} / G_{R 3}$ & $L_{3}, L_{7}, \ldots, L_{N-1} / R_{3}, R_{7}, \ldots, R_{N-1}$ \\
$G_{L 4} / G_{R 4}$ & $L_{4}, L_{8}, \ldots, L_{N} / R_{4}, R_{8}, \ldots, R_{N}$ \\
\hline
\end{tabular}

be deemed as a signal group unit to classify all sensor nodes in Figure 1, as shown in Table 1.

In this table, to distinguish the sensor nodes and signal groups at both sides of train, $L_{i}$ denotes the $i$ th sensor node at the left side, $R_{i}$ represents the $i$ th sensor node at the right side, $G_{L k}$ refers to the $k$ th signal group at the left side, and $G_{R k}$ indicates the $k$ th signal group at the right side.

The on-line inspection of rail defects requires all sensor nodes to collect data by equidistant space sampling, that is, sample once when the wheels turn for $1 / z$ round. Let the driving distance of train be $s$, the signal collected by $L_{i}$ sensor node can be represented by $l_{i}(s)$, and the signal collected by $R_{i}$ sensor node is denoted by $r_{i}(s)$.

\section{Rail Defect Inspection Based on the Two-Dimensional Impact Reconstruction}

For the signals collected by a single-sensor node as mentioned above, the impacts caused by rail defects and the noises of locomotive running gear are not periodic, and those caused by rail joints and defects of locomotive running gear are periodic, so it is difficult to distinguish periodic and aperiodic impacts of tracks and vehicles. To avoid limitations of single-sensor information studying, a two-dimensional impact reconstruction method is put forward from the perspective of multisensor information fusion $[19,20]$. This method is built on the wireless sensor network for on-line inspection of rail defects with the signal groups for study (see Table 1 for division of signal groups) and based on the multisensor information fusion of two-dimensional images. Its processing includes multisensor information preprocessing, shifting time-frequency analysis, and time-frequency spectrum image analysis and image fusion.

The wheel diameter is $D$, the full length of train (without external force, the distance of the internal side of coupler knuckle when the couplers at both ends are in closed position) is $S_{1}$, and the distance between rail joint gaps is $S_{2}$. For the convenience of explaining the two-dimensional impact reconstruction process, the signal group $G_{L k}$ is taken as an example for detailed discussion (the two-dimensional impact reconstruction of the signal group $G_{R k}$ is similar and thus omitted). The process is as follows.

3.1. Multisensor Information Preprocessing. Multisensor information preprocessing mainly comprises equidistant space resampling and threshold processing. First of all, all sensor node signals in the signal group for nonequidistant space sampling (hereinafter referred to as group signals) need the wheel speed collected by the speed sensor to make 

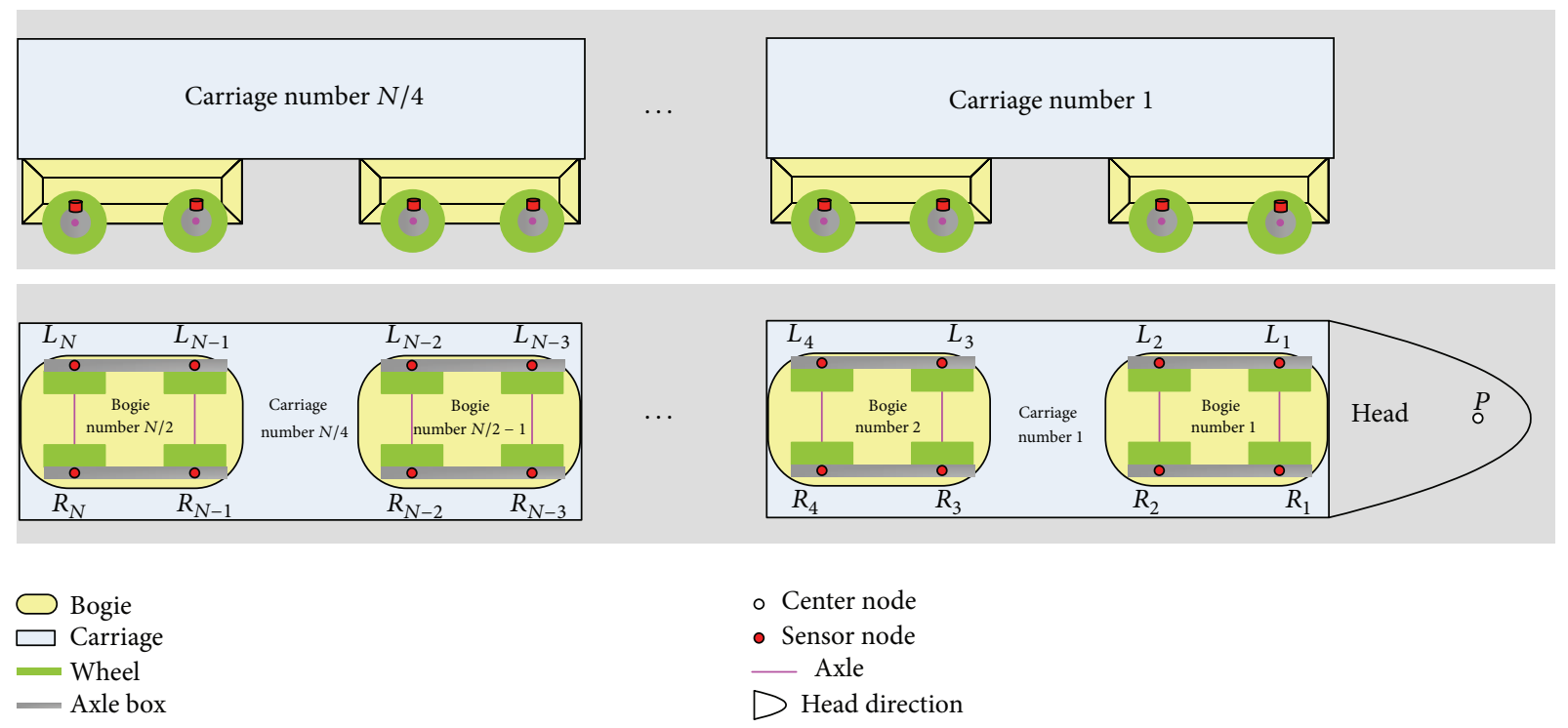

FIGURE 1: Node distribution shown in vertical and front views of the train.

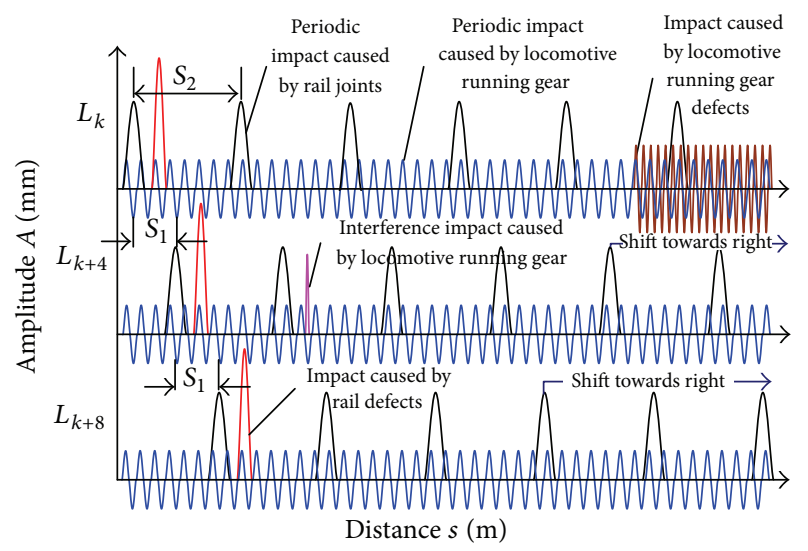

FIGURE 2: Sketch map of preprocessed inner group signal in space domain.

equidistant space resampling of signals and convert time domain signals to space domain signals and corresponding physical concepts such as time domain, period, and frequency to space domain, spatial period, and spatial frequency. Then, signals of sensor nodes for equidistant space sampling have been conducted the threshold processing, that is, artificially setting a threshold to filter the noise interference information the observer does not feel interested in.

Figure 2 shows the waveforms of space domains obtained after preprocessing of signals gathered by the nodes $L_{k}$, $L_{k+4}$, and $L_{k+8}$ in the signal group $G_{L k}$, denoted by $l_{k}(s)$, $l_{k+4}(s)$, and $l_{k+8}(s)$, respectively. In order to simplify problem and better emphasize the key point, we suppose that $l_{k}(s)$, $l_{k+4}(s)$, and $l_{k+8}(s)$ all contain the spatial periodic impact (pulse type) caused by rail joints, spatial nonperiodic impact (pulse type) caused by rail defects, and spatial periodic impact (harmonic type) caused by locomotive running gear and noise signal (not drawn in the figure). Besides, let $l_{k}(s)$ contain the spatial periodic impact (pulse type) caused by the defect of locomotive running gear and let $l_{k+4}(s)$ contain the spatial interference impact (pulse type) caused by locomotive running gear.

3.2. Shifting Time-Frequency Analysis. Considering that the train in operation is influenced by various uncertain factors, the vertical vibration signals are obviously non-Gaussian and nonstationary. In the light of pertinence of signals in the group, for extracting the rail failure feature information, the shifting time-frequency analysis method is proposed. It is an improvement for the modern time-frequency analysis aiming at extracting of rail failures. This method aligns and shifts the preprocessed signals in groups in space domain to fully dig out the relevancy of signals in the groups and then acquires their spatial time-frequency spectrum by the modern timefrequency analysis, as shown in Figure 3.

Figure 3(a) describes the aligning and shifting of space domain signals shown in Figure 2. Firstly, it determines a benchmark, that is, the spatial periodic impact caused by rail joints in the signal $l_{k}(s)$ collected by the first node $L_{k}$ in the signal group $G_{L k}$ as the benchmark. Secondly, the phase shifts towards left to return to zero, to be exact, the signal $l_{i}(s)$ collected by the node $L_{i}$ is left shifted by $S_{1}(i-k) / 4$ so that all node information in the signal group $G_{L k}$ is synchronized in distance. Finally, the phase shifts towards right to realize the spatial periodicity property; namely, the signal $l_{i}(s)$ is right shifted by $m S_{2}(i-k) / 4$ so that the phase difference of spatial periodic impact caused by rail joints of signals $l_{i}(s)$ collected by the nodes is $m S_{2}$ and that of spatial nonperiodic impact caused by rail defects is also $m S_{2}$.

Figure 3(b) shows the spatial time-frequency spectrum of the signal shown in Figure 3(a).

In the following, we take short-time Fourier transform as an example of the modern time-frequency analysis, to deduce the formula of shifting time-frequency analysis. 


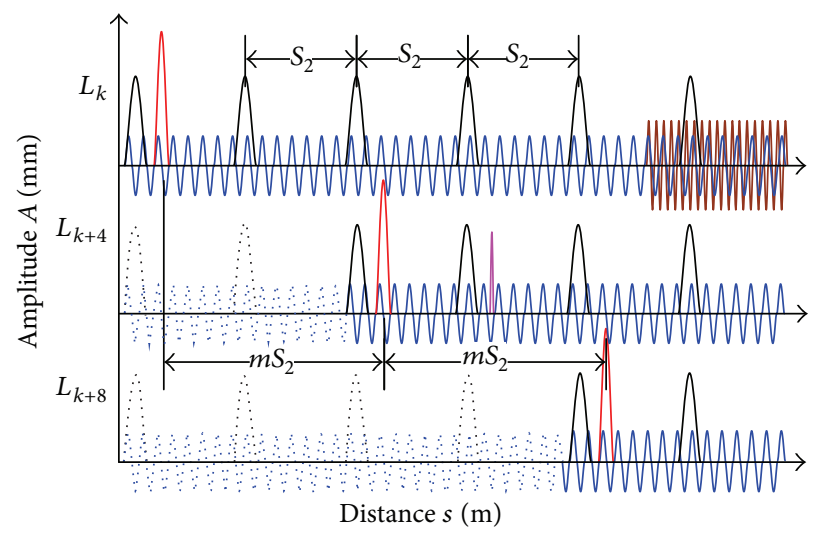

(a) Aligning and shifting of space domain signals

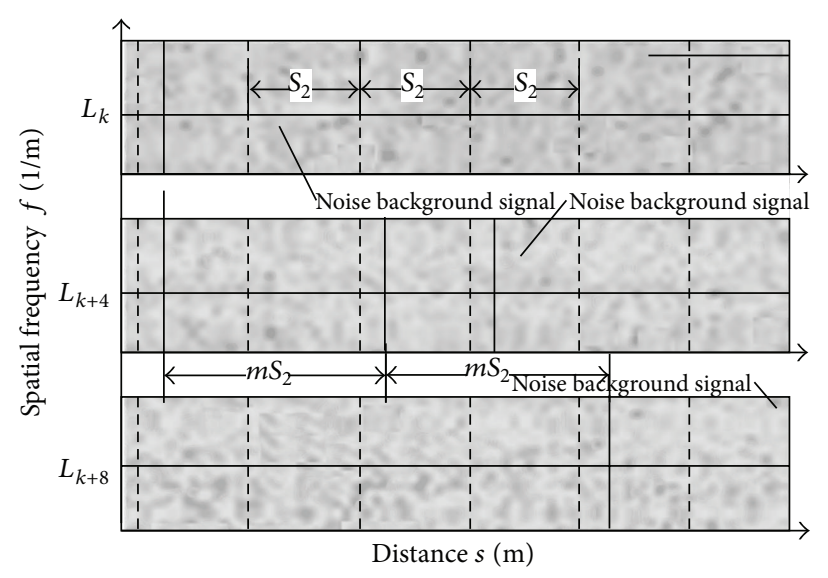

(b) Time-frequency analyzing and processing

FIGURE 3: Sketch map of shifting time-frequency analyzing and processing.

After $l_{i}(s)$ is processed by using shifting time-frequency analysis, $L_{i}(s, f)$ can be obtained, as shown in

$$
\begin{aligned}
L_{i}(s, f)=\int_{-\infty}^{+\infty} l_{i}\left(s+\frac{\left(S_{1}-m S_{2}\right)(i-k)}{4}\right) \\
\times h(s-\tau) e^{-j 2 \pi f \tau} d \tau, \quad k=1,2,3,4 ; \\
i=k, k+4, k+8, \ldots, N+k-4 ; \quad m=2,3, \ldots,
\end{aligned}
$$

where $L_{i}(s, f)$ is the spatial time-frequency spectrum after shifting time-frequency analysis and $h(s)$ represents the window function.

In the equation, the value of $m$ must be the integral number greater than or equal to 2 , or else the impact feature information may be blurred. Consider the following.

(a) If $m$ is equal to 0 , nonperiodic impacts caused by rail defects of nodes in the group have the same phase, which will blank the nonperiodic impacts after subsequent image fusion and result in loss of impact.

(b) If $m$ is equal to 1 , although nonperiodic impacts caused by rail defects of nodes in the group have the same phase difference between each other, after the subsequent image fusion, the nonperiodic impacts caused by rail defects have the same periodic features with the periodic impacts caused by rail joints, thus leading to impact overlap.

(c) If $m$ is not an integral number, the periodic impacts caused by rail joints and locomotive running gear of nodes in the groups will have phase difference, and after the subsequent image fusion, the interference information may be introduced and the false impact may exist.

3.3. Time-Frequency Spectrum Image Analysis. In order to achieve better fusion performance of images in the subsequent "image fusion processing," the spatial time-frequency spectrum obtained after shifting time-frequency analysis still needs impact features extraction and node color labeling for further time-frequency spectrum image analysis, as shown in Figure 4.

3.3.1. Impact Feature Extraction. Impact feature extraction refers to a process in which the image grey scale processing technique is used to remove the noise background signals in order to highlight the impact signals. As the impact signals to be extracted from the spatial time-frequency spectrum contain the noise background signals, it is really necessary to perform the impact feature extraction. Its principle can be described as follows: firstly, the spatial time-frequency spectrum image is given statistics using the grey scale histogram, to find out the threshold between the impact signals and noise background signals, the dark grey corresponding to the impact signals and the light grey corresponding to the noise background signals. Then, using the captured threshold for gray threshold transform, the spatial time-frequency spectrum image is converted into the black and white binary images, the white corresponding to the noise background signals and the black corresponding to the impact signals. In the following, the impact feature extraction is in detail introduced with the schematic diagram.

The numeric area $[a, b]$ of the spatial time-frequency spectrum $L_{i}(s, f)$ as shown in Figure 3(b) turns into $[0,255]$ when mapped onto the grey space. If the grey scale histogram is used for deciding the threshold $\lambda_{i}$, then the spectral value of the spatial time-frequency spectrum to which the threshold corresponds is $\sigma_{i}=(b-a) \lambda_{i} / 255+a$. After gray threshold transforming, all points which lie in $L_{i}(s, f) \geq \sigma_{i}$ on the spatial time-frequency spectrum are made black, and other points are made white, and the spatial time-frequency spectrum image is then converted into the black and white binary image, as shown in Figure 4(a). It can be seen that the noise background signal contained in the nodes of the signal group $G_{L k}$ is removed, and all impact signals are highlighted; thus the impact feature extraction is realized. 


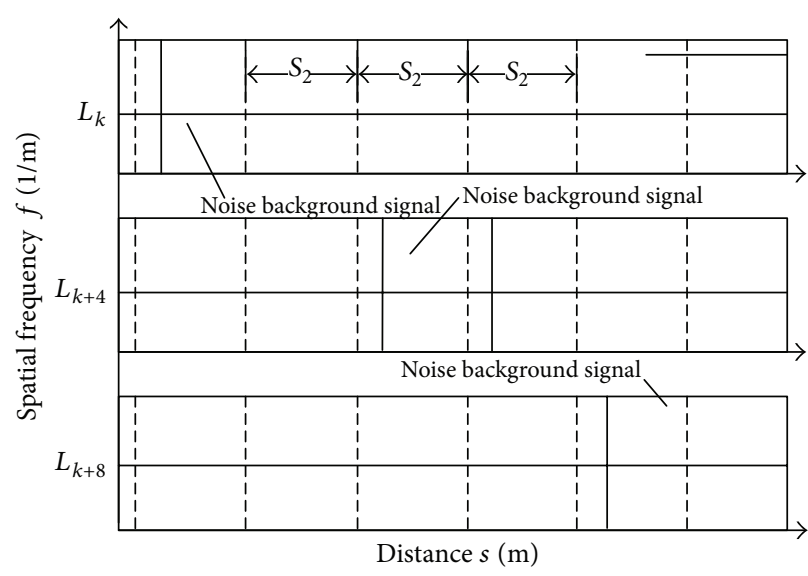

(a) Impact feature extracting

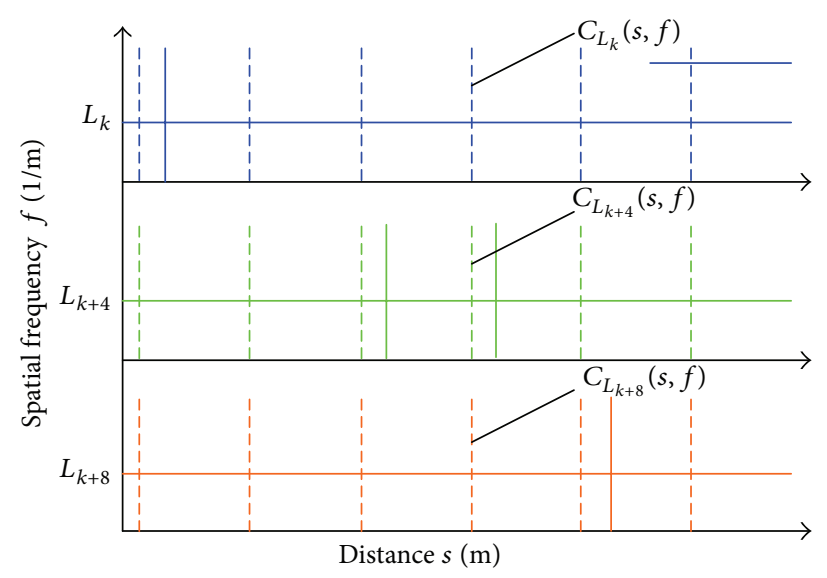

(b) Node color labeling

FIGURE 4: Sketch map of time-frequency spectrum image processing.

3.3.2. Node Color Labeling. Node color labeling refers to a process in which the blanking technique is used to remove the periodic impact signals from the impact signals arising from all kinds of factors, so as to extract the impact signals resulting from the rail defects. As the impact signals extracted are quite complex in terms of make-up, and, in particular, the periodic impact signals resulting from the locomotive running gear and rail joints, which are widely distributed in the whole space domain, may cause great effect on judgment of the impacts caused by the rail defects, it is really necessary to perform the node color labeling. If the spatial time-frequency spectrum of the signal of the node $L_{i}$ within the signal group $G_{L k}$ is $L_{i}(s, f)$, then the spatial time-frequency spectrum of the node $L_{i}$ can be labeled as follows: with the color labeling method as shown in (2) and its constraint equation (3), the different colors $C_{L i}(s, f)$ are used to label the spatial time-frequency spectrum image of the signal of the node $L_{i}$, as shown in Figure 4(b), so that the impact signals of the nodes at the same spatial position on the spectrum image are converted into the white background after the subsequent image fusion processing so as to blank the periodic impact signals. Consider

$$
\begin{gathered}
C_{L i}(s, f)= \begin{cases}\operatorname{RGB}(255,255,255) & L_{i}(s, f)<\sigma_{i} \\
\operatorname{RGB}\left(r_{i}, g_{i}, b_{i}\right) & L_{i}(s, f) \geq \sigma_{i},\end{cases} \\
i=k, k+4, k+8, \ldots, N+k-4,
\end{gathered}, \begin{array}{ll}
\sum_{i=k}^{N+k-4} r_{i}=255 \quad 0 \leq r_{i} \leq 255, \\
\sum_{i=k}^{N+k-4} g_{i}=255 \quad 0 \leq g_{i} \leq 255, \\
\sum_{i=k}^{N+k-4} b_{i}=255 \quad 0 \leq b_{i} \leq 255,
\end{array}
$$

$$
\begin{aligned}
& \operatorname{RGB}\left(r_{i}, g_{i}, b_{i}\right) \neq \operatorname{RGB}\left(r_{j}, g_{j}, b_{j}\right) \quad i \neq j, \\
& k=1,2,3,4 ; \quad i, j=k, k+4, k+8, \ldots, N+k-4,
\end{aligned}
$$

where $L_{i}(s, f)$ is the spatial time-frequency spectrum of the signals of the node $L_{i}$ within the signal group $L_{i}(s, f), r_{i}, g_{i}$, and $b_{i}$ are the color values of the components of the tricolor RGB, respectively, $C_{L i}(s, f)$ is the image color of the labeled $L_{i}(s, f)$, and $\sigma_{i}$ is the spectral value of the spatial timefrequency spectrum to which the threshold $\lambda_{i}$ decided by using the grey scale histogram corresponds.

Equation (2) defines the method of node color labeling, and (3) is its constraint. The first three equations in (3) are the necessary conditions for blanking, to make sure periodic impact caused by rail joints at each node within the group and periodic impact caused by locomotive running gear are blanked to white in subsequent "image fusion processing." The last inequation in (3) is a constraint designed to prevent the subsequent "image fusion processing" from causing the node code information loss and resulting in multiple impacts lines with the same color.

3.4. Image Fusion Processing. The multisensor information preprocessing, shifting time-frequency analysis, and timefrequency spectrum image analysis mentioned above are the first-phase preparations for the two-dimensional impact reconstruction method, and they convert the signals from one-dimensional space domain signals (as shown in Figure 2) into two-dimensional image signals (as shown in Figure 4). To explore as much relevancy between the multisensor information as possible and realize the feature extraction of the rail defects, it is necessary to perform the image fusion processing for the two-dimensional image signals derived from the processing above. And the principle can be described as follows: according to the pixel point color fusion algorithm as shown in (4), the spectrum (as shown in Figure 5) is obtained by fusing all the spatial time-frequency spectrum images of the node signals within the signal group 


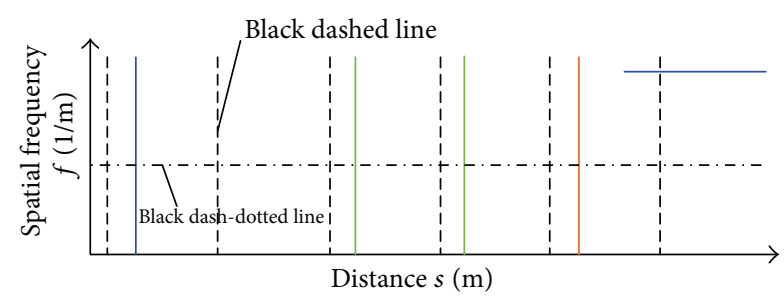

$$
\begin{aligned}
& -L_{k} \\
& -L_{k+4} \\
& -L_{k+8}
\end{aligned}
$$

FigURE 5: Sketch map of image fusion processing.

derived from the time-frequency spectrum image analysis, so as to realize the two-dimensional image reconstruction of the impact signals resulting from the rail defects. Consider

$$
\begin{gathered}
C_{G_{L k}}(s, f)=\operatorname{RGB}\left(x_{i}, y_{i}, z_{i}\right), \\
x_{i}=255-\sum_{i=k}^{N+k-4}\left(255-r_{i}\right) \% 255, \\
y_{i}=255-\sum_{i=k}^{N+k-4}\left(255-g_{i}\right) \% 255, \\
z_{i}=255-\sum_{i=k}^{N+k-4}\left(255-b_{i}\right) \% 255, \\
k=1,2,3,4 ; \quad i=k, k+4, k+8, \ldots, N+k-4,
\end{gathered}
$$

where $r_{i}, g_{i}$, and $b_{i}$ are the color values of the components of the tricolor RGB, respectively, and $C_{G_{L k}}(s, f)$ is the color of the two-dimensional impact reconstruction image fused by $C_{L i}(s, f)$ of all nodes within the signal group $G_{L k}$ on the left side of the train.

Equation (4) has the physical meaning as follows: when several images whose grey value is 255 are fused with one image whose grey value is $h(0 \leq h \leq 255)$, the grey value of the image derived from such fusion will be $h$, and when several images whose grey value is 255 are fused, the grey value of the image derived from such fusion will be 255 .

After substituting (2) and (3) into (4), the images of the nodes as defined in Figure 4(b) are fused, and the results of fusion are as shown in Figure 5. The figure shows that the periodic impacts caused by the rail joints and locomotive running gear (for the convenience of understanding and reading, in Figure 5, they are, respectively, expressed by the black dashed line and dash dot line, which are actually white dashed line and white solid line) are blanked after image fusion, which is because the above periodic impacts occur at the same place at each node within the signal group $G_{L k}$ and the color labeling at each node meets (3); then the color turns to RGB $(255,255,255)$ after fusion. Meanwhile, the aperiodic impacts only occur at particular node within signal group $G_{L k}$ and the color at such node remains the same after fusion; as a consequence, the aperiodic impacts resulting from defects caused by the rail and the locomotive running gear are highlighted, and the node code information is retained by labeled colors.

After image fusion, the impact signals caused by the rail defects have been two-dimensionally reconstructed so as to make them different from other impact signals. The twodimensional reconstructed impact signals caused by the rail defects have the following features.

(a) Impact signals cover all nodes, and N/4 impact lines appear.

(b) Impact lines vary in color, and all of them become white after image fusion.

(c) The period of the impact lines within partial space is $m S_{2}$.

Based on the feature information above, it can be judged whether or not there are rail defects, and if there are rail defects, they can also be located. When the three characteristics above occur, the rail can be judged defective and the defect is where the impact line at the first node $L_{k}$ in signal group $G_{L k}$ is located.

\section{Application Analysis}

The two-dimensional impact reconstruction method focuses on multisensor information as study object while the modern time-frequency method takes single-sensor information as object, so the former is more suitable to inspect rail defects. To validate the effectiveness of the two-dimensional impact reconstruction method, short-time Fourier transform method and the two-dimensional impact reconstruction method have been used into the comparison and analysis of vibration data of a sixteen-carriage train.

Given that the train comprises 16 carriages, its whole length $S_{1}$ is $25,175 \mathrm{~mm}$, and its wheel diameter $D$ is $915 \mathrm{~mm}$. The installation arrangement of the vibration impact sensors and speed sensors is as shown in Figure 1 (according to distribution of nodes in the signal group $G_{L 1}, 16$ vibration impact sensors are installed above the axle boxes of the 16 carriages, separately). The data is captured by each vibration impact sensor once as the wheel rotates $1 / 2,000$ cycle, and totally 16 groups of data are acquired (the train is running on the track whose length of rail $S_{2}$ is $25,000 \mathrm{~mm}$ while acquiring data).

Figure 6(a) shows the space domain waveform of vertical vibration signals at the node $L_{1}$. Figure 6(b) shows the result obtained after the vertical vibration signal is processed by short-time Fourier transform method (select Gaussian windowed short-time Fourier transform, and the size of Gaussian window is $12.8 \mathrm{~mm}$ ). As shown in Figure 6(b), both the periodic impact (as shown by A in Figure 6(b)) and the aperiodic impact (as shown by B in Figure 6(b)) can be extracted effectively by short-time Fourier transform; however, it is difficult to tell whether B is caused by rail defects, for the reason that the aperiodic impact might be caused by noise, locomotive running gear, and so forth.

Figure 7 shows the detailed process in which the proposed two-dimensional impact reconstruction method is adopted to extract the rail defect features from the 16 groups 


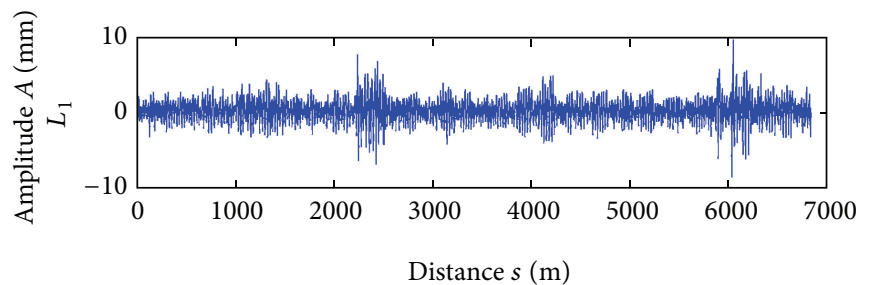

(a)

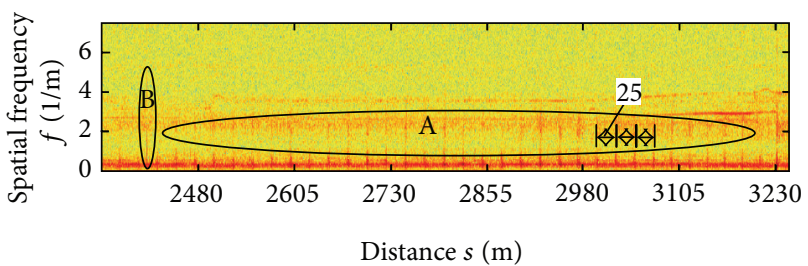

(b)

FIGURE 6: (a) Space domain wave of vertical vibration signals at the node $L_{1}$ and (b) spatial time-frequency spectrum after short-time Fourier transform.

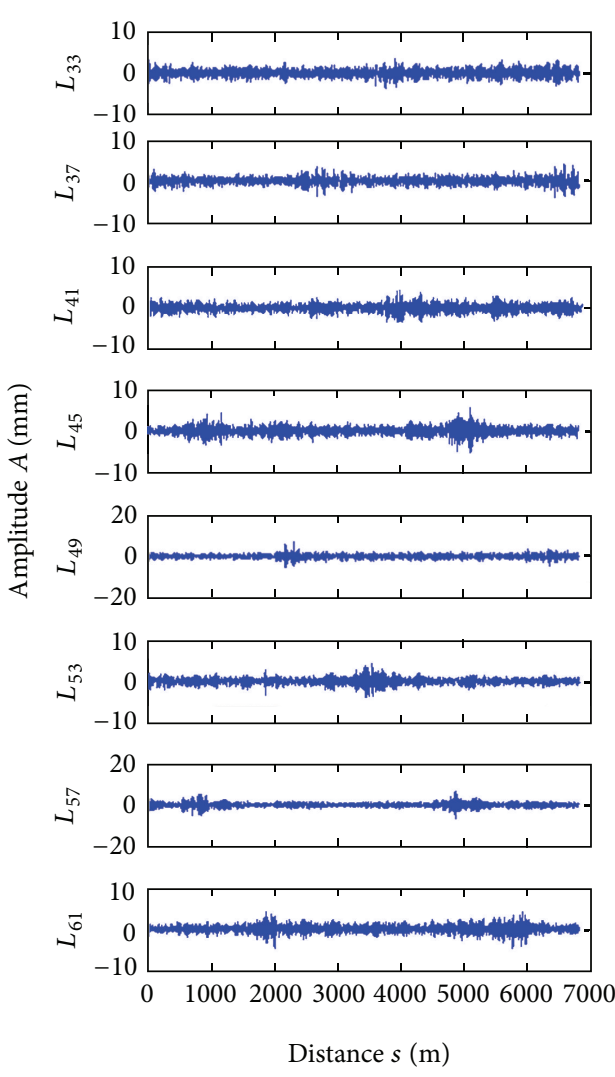

(a)

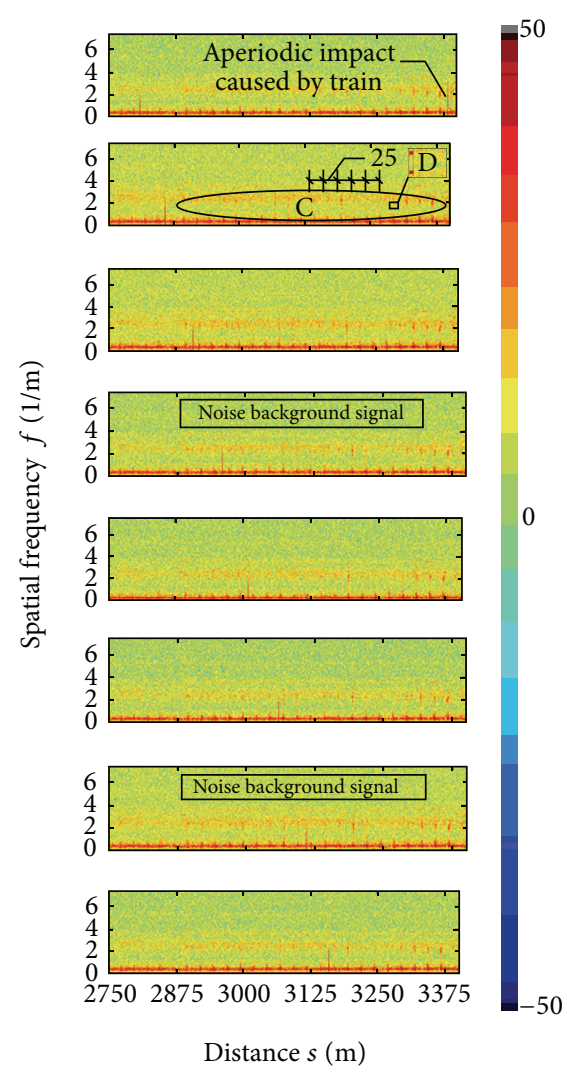

(b)

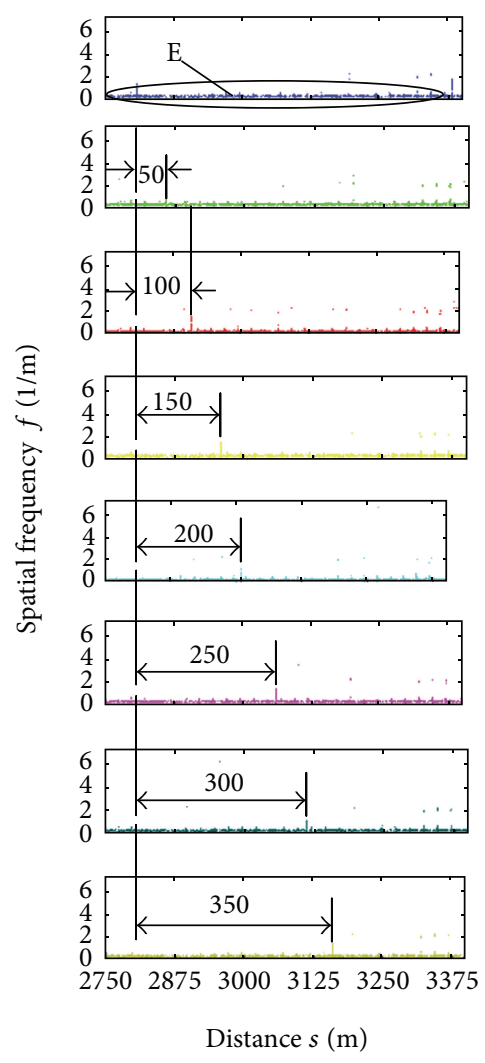

(c)

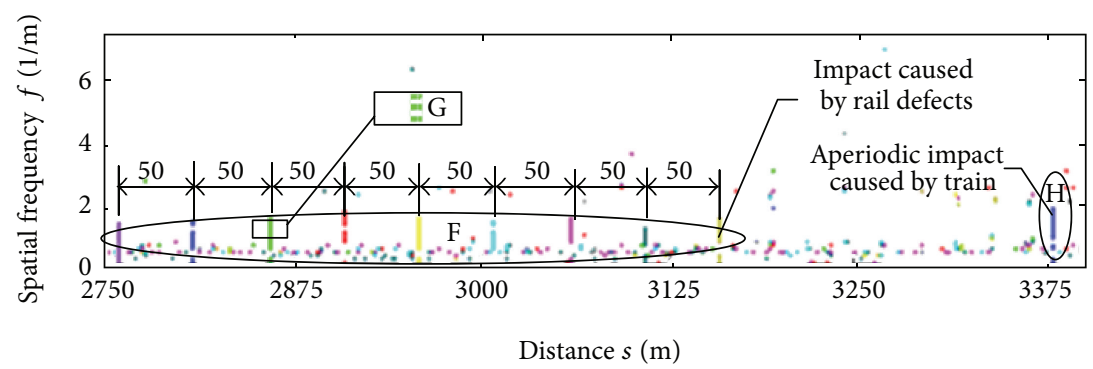

(d)

FIGURE 7: (a) Space domain wave of multisensor signal after preprocessing, (b) spatial time-frequency spectrum after shifting time-frequency analysis, (c) spatial time-frequency spectrum image after time-frequency spectrum image analysis, and (d) impact feature chart after image fusion processing. 
of data corresponding to the signal group $G_{L 1}$ (only the last 8 groups of data are shown in the figure). Plots of the preprocessed 16 groups of original data in space domain are shown in Figure 7(a). Figure 7(b) shows the spatial time-frequency spectrum derived from the shifting time-frequency analysis (let $m=2$, select Gaussian windowed short-time Fourier transform as the modern time-frequency analysis method, and the size of Gaussian window is $12.8 \mathrm{~mm}$ ). Figure $7(\mathrm{c})$ shows the spatial time-frequency spectrum image derived from the time-frequency spectrum image analysis with the threshold $\sigma=0.46$. Figure 7(d) shows the impact feature chart derived from the image fusion.

It can be seen from Figures 7(c) and 7(d) that the noise background signals in Figures $7(\mathrm{a})$ and $7(\mathrm{~b})$ are largely removed, and the impact signals are highlighted. In Figures 7(b) and 7(c), the spatial frequency components of impact sequence whose period is $0.04 \mathrm{~m}^{-1}$ (corresponding to the periodic impacts arising from the rail joints, as shown by $\mathrm{C}$ and $\mathrm{D}$ in Figure $7(\mathrm{~b})$ ) are always seen at $25 \mathrm{~m}$ intervals across the whole travel, and the signals having the fixed spatial frequency of $0.35 \mathrm{~m}^{-1}$ across the whole travel (corresponding to the periodic impacts arising from the rotation frequency of the train wheel, as shown by E in Figure 7(c)) are all blanked to some extent, as shown in Figure 7(d). The spatial frequency components of impact sequence whose period is $0.02 \mathrm{~m}^{-1}$ (corresponding to the aperiodic impacts arising from the rail failure around $2,410 \mathrm{~m}$, as shown by $\mathrm{F}$ and $\mathrm{G}$ in Figure 7(d)) are obviously seen at $50 \mathrm{~m}$ intervals across the partial travel, and a spatial wide band signal (corresponding to the aperiodic impacts arising from the train, as shown by $\mathrm{H}$ in Figure 7(d)) is seen around 3,395 m.

The application above shows that the two-dimensional impact reconstruction method is able to remove the noise background signals, highlight the aperiodic impact signals arising from the factors such as rail defects (as shown in Figure $7(\mathrm{~d})$ ), and allow the aperiodic impact signals resulting from rail defects to undergo the two-dimensional reconstruction to be different from the periodic impact signals within the partial space domain, so as to extract the impact features caused by the rail defects and realize the on-line inspection and location of the rail defects. In conclusion, twodimensional impact reconstruction method is an effective method to extract track defects.

\section{Conclusion}

The two-dimensional impact reconstruction method integrates the wireless sensor network technique, inspection technique, information fusion technique, signal processing technique, and image processing technique and mainly involves such processes as multisensor information preprocessing, shifting time-frequency analysis, time-frequency spectrum image analysis, and image fusion processing. It can be used to make on-line analysis of the vertical vibration signals of the train as it is running, extract the aperiodic impact features caused by the rail defects effectively, and realize the on-line inspection and location of rail defects.

The two-dimensional impact reconstruction method mainly possesses the following characteristics. (a) It uses the image grey scale processing technique, so it can remove the noise background signals and highlight all kinds of impact signals well.

(b) It uses the blanking technique, so it can remove the periodic impact signals from the impact signals well, and make the impact signals arising from the rail defects easily extracted.

(c) It can convert the aperiodic impacts arising from the rail defects into periodic ones within a certain partial space.

(d) It can on-line extract the impact features arising from the rail defects as the train is running and locate the rail defects.

The method also deserves further investigation on the following aspects.

(a) Considering that the proposed method involves shifting time-frequency analysis, image processing, and so forth, the processing speed is limited for the large amount of calculation. Although presently it can be used to give on-line inspection of the rail defects, it requires further study in terms of real-time inspection.

(b) Although it can remove the periodic impact signals, the impact signals still contain other aperiodic impact signals other than those arising from the rail defects (interference impacts caused by the locomotive running gear, impacts caused by defects of the locomotive running gear, etc.); further study is needed in this regard.

\section{Conflict of Interests}

The authors declare that there is no conflict of interests regarding the publication of this paper.

\section{Acknowledgments}

This work was supported in part by the Key National Natural Science Foundation of China (Grant no. 61134002), and the National 863 plans projects (Grant no. 2011AA110501).

\section{References}

[1] E. G. Berggren, M. X. D. Li, and J. Spännar, "A new approach to the analysis and presentation of vertical track geometry quality and rail roughness," Wear, vol. 265, no. 9-10, pp. 1488-1496, 2008.

[2] M. J. M. M. Steenbergen, "Quantification of dynamic wheelrail contact forces at short rail irregularities and application to measured rail welds," Journal of Sound and Vibration, vol. 312, no. 4-5, pp. 606-629, 2008.

[3] G. Lombaert and G. Degrande, "Ground-borne vibration due to static and dynamic axle loads of InterCity and high-speed trains," Journal of Sound and Vibration, vol. 319, no. 3-5, pp. 1036-1066, 2009. 
[4] J. Vega, A. Fraile, E. Alarcon, and L. Hermanns, "Dynamic response of underpasses for high-speed train lines," Journal of Sound and Vibration, vol. 331, no. 23, pp. 5125-5140, 2012.

[5] X. Y. Liu and W. M. Zhai, "Analysis of vertical dynamic wheel/rail interaction caused by polygonal wheels on highspeed trains," Wear, vol. 314, no. 1-2, pp. 282-290, 2014.

[6] Q. Li and S. Ren, "A visual detection system for rail surface defects," IEEE Transactions on Systems, Man and Cybernetics Part C: Applications and Reviews, vol. 42, no. 6, pp. 1531-1542, 2012.

[7] M. Guerrieri, G. Parla, and D. Ticali, "Mono and stereoscopic image analysis for detecting the transverse profile of worn-out rails," Procedia-Social and Behavioral Sciences, vol. 53, no. 1-2, pp. 611-621, 2014.

[8] L. S. Wu, Y. W. Li, H. W. Chen et al., "Research on rail defects automatic detection technology based on image region partition," Laser Infrared, vol. 42, no. 5, pp. 594-599, 2012.

[9] K. Xu, C. Yang, P. Zhou, and J. Liang, "3D detection technique of surface defects for steel rails based on linear lasers," Journal of Mechanical Engineering, vol. 46, no. 8, pp. 1-5, 2010.

[10] Y. Fan, S. Dixon, R. S. Edwards, and X. Jian, "Ultrasonic surface wave propagation and interaction with surface defects on rail track head," NDT and E International, vol. 40, no. 6, pp. 471477, 2007.

[11] S. Mariani, T. Nguyen, R. R Phillips et al., "Noncontact ultrasonic guided wave inspection of rails," Structural Health Monitoring, vol. 12, no. 5-6, pp. 539-548, 2013.

[12] B. M. Hopkins and S. Taheri, "Track health monitoring using wavelets," in Proceeding of the ASME Rail Transportation Division Fall Technical Conference (RTDF '10), pp. 9-15, October 2010.

[13] F. Lanza di Scalea and J. McNamara, "Measuring highfrequency wave propagation in railroad tracks by joint timefrequency analysis," Journal of Sound and Vibration, vol. 273, no. 3, pp. 637-651, 2004.

[14] A. Caprioli, A. Cigada, and D. Raveglia, "Rail inspection in track maintenance: a benchmark between the wavelet approach and the more conventional Fourier analysis," Mechanical Systems and Signal Processing, vol. 21, no. 2, pp. 631-652, 2007.

[15] L. Law, J. H. Kim, W. Y. H. Liew, and S. Lee, "An approach based on wavelet packet decomposition and HilbertHuang transform (WPDHHT) for spindle bearings condition monitoring," Mechanical Systems and Signal Processing, vol. 33, pp. 197-211, 2012.

[16] S. Jiang, Q. Fu, and Z. Wen, "Application of wavelet transform to obtain track static power spectrum density," Journal of Traffic and Transportation Engineering, vol. 4, no. 2, pp. 33-39, 2004.

[17] G. D. Zhou and T. H. Yi, "Recent developments on wireless sensor networks technology for bridge health monitoring," Mathematical Problems in Engineering, vol. 2013, Article ID 947867, 33 pages, 2013.

[18] K. Deng and Z. Liu, "Target tracking with dynamic clusters in wireless sensor network," Acta Armamentarii, vol. 29, no. 10, pp. 1197-1202, 2008.

[19] J. Zhang, B. Wang, J. Di, H. Yu, and B. Lu, "Research on information fusion for sensors multiple fault diagnosis," Proceedings of the Chinese Society of Electrical Engineering, vol. 27, no. 16, pp. 104-108, 2007.

[20] M. S. Safizadeh and S. K. Latifi, "Using multi-sensor data fusion for vibration fault diagnosis of rolling element bearings by accelerometer and load cell," Information Fusion, vol. 18, pp. 1-8, 2014. 


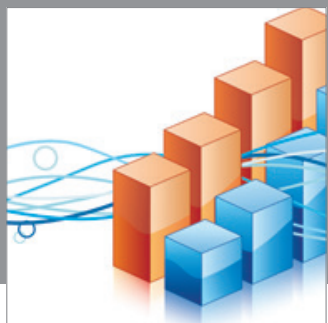

Advances in

Operations Research

mansans

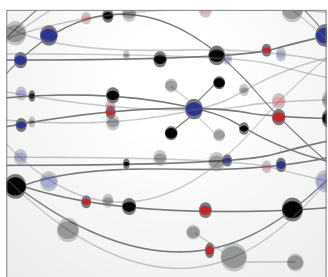

The Scientific World Journal
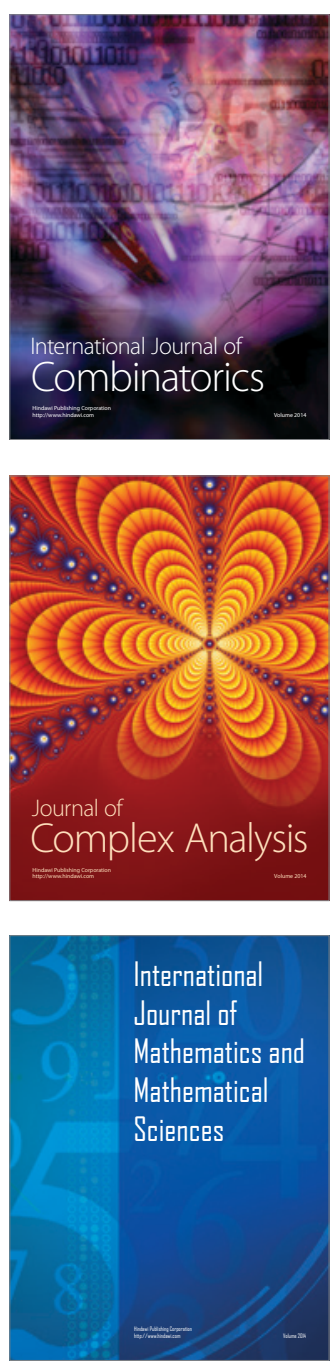
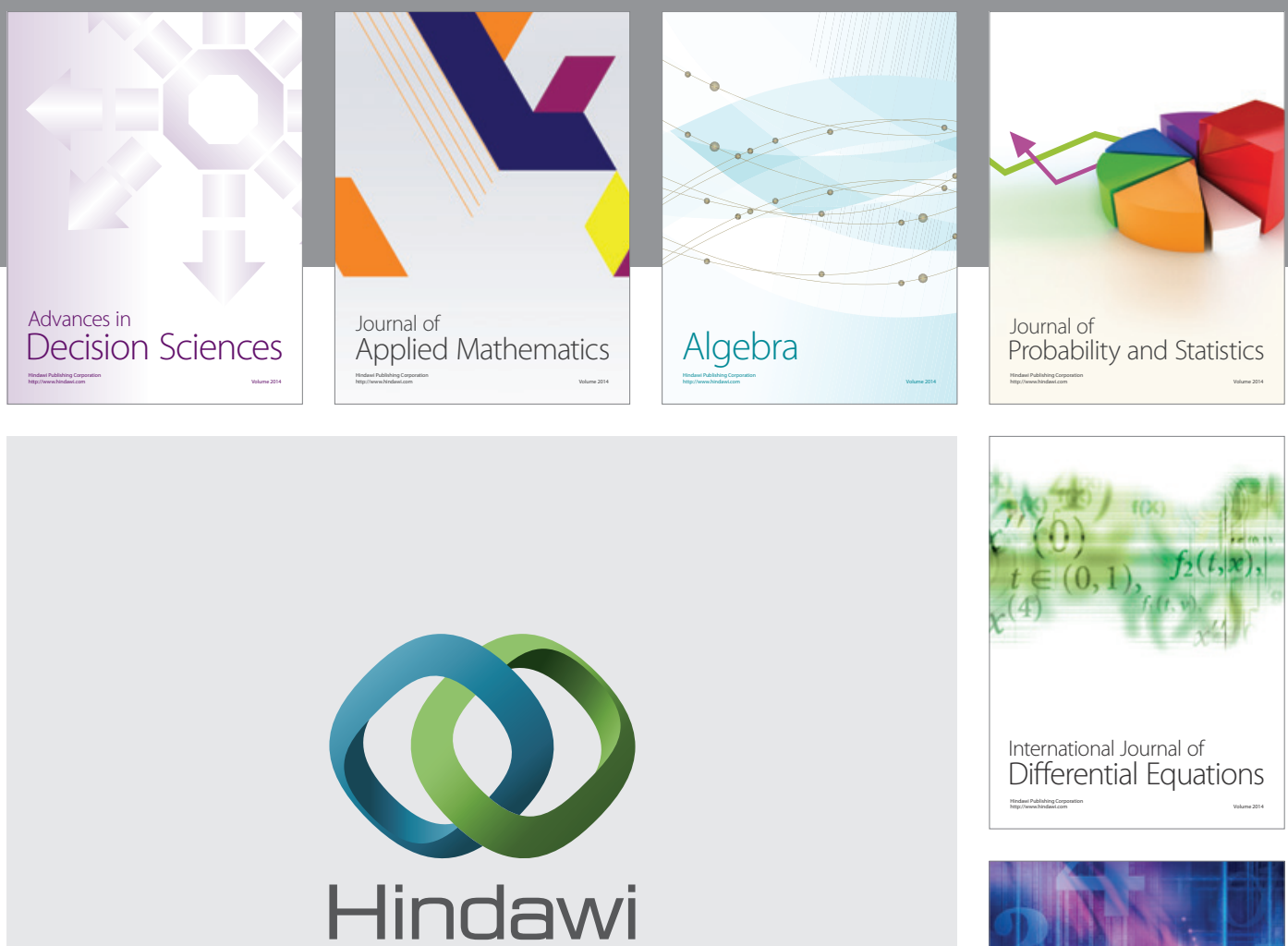

Submit your manuscripts at http://www.hindawi.com
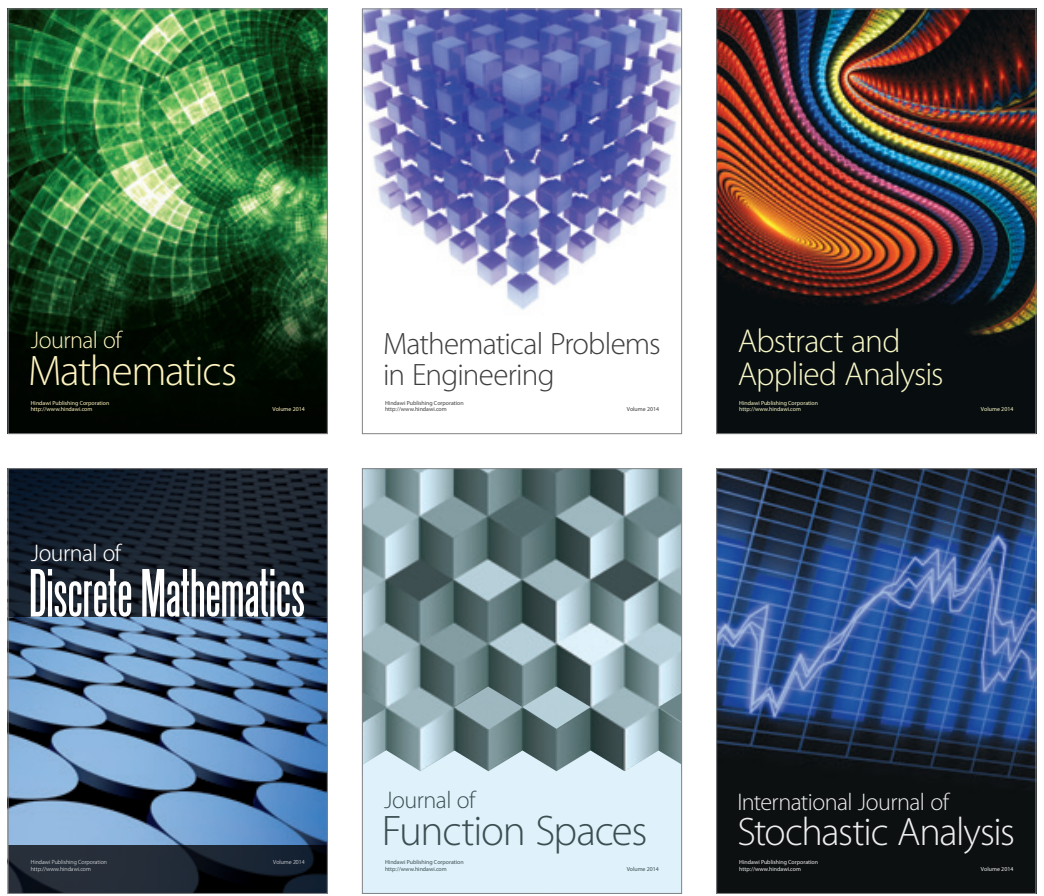

Journal of

Function Spaces

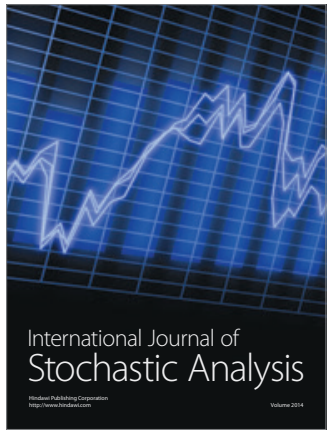

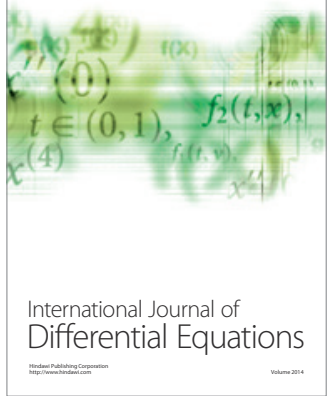
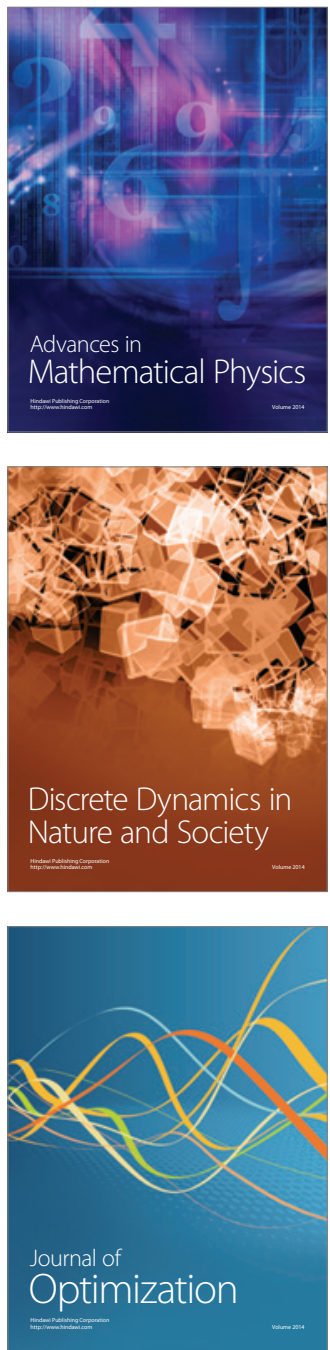